\title{
Simultaneous PLIF/PIV measurements of pulsating and heated coaxial jets in a turbulent channel flow
}

\author{
J. Klinner ${ }^{1}$, C. Willert ${ }^{1}$, W. Förster ${ }^{1}$, M. Beversdorff ${ }^{1}$ and V. Mayer $^{2}$ \\ ${ }^{1}$ Institute of Propulsion Technology, German Aerospace Center (DLR), \\ 51170 Köln, Germany, joachim.klinner@dlr.de
}

${ }^{2}$ Daimler AG, D-70546 Stuttgart, Germany

\section{Introduction}

This contribution describes a generic mixing experiment that is designed to provide relevant data of turbulence induced mass transfer to validate scalar transport simulations. The measurements are performed in a small scale wind tunnel as outlined in Fig. 1. The mixing port is placed at a third of the test section length and consists of a $90^{\circ}$ bend. The bend exit is aligned coaxially to the channel's center line. Three modes of operation are investigated at constant mass flow rates: (A) isothermal steady jet injection, $R e_{d}=32000$ (c.f. [1]), (B) pulsed isothermal injection with a pulsation frequency of $66 \mathrm{~Hz}$ and a Strouhal number of 0.08 and (C) steady injection at elevated jet temperatures of $\Delta \mathrm{T}=50 \mathrm{~K}$ and $\Delta \mathrm{T}=100 \mathrm{~K}$.

Ensemble-averages of velocity, mixing fraction, Reynolds stresses and turbulent mass flux are determined at certain positions downstream of the nozzle using sets of up to 3600 PLIFPIV recordings. During pulsed operation, phase angles are simultaneously monitored with each single shot to enable a posteriori phase sorting and phase averaging.

The measurement uncertainty of each velocity component is estimated from uncertainties of the displacement detection. The stability of the initial acetone tracer concentration over time is assessed from fluorescence measurements of unmixed fluid. The systematical error due to temperature dependency of fluorescence quantum yield is discussed and partially corrected. The statistical error of measured quantities over the number of samples is assessed in exemplary regions within the shear layers of the mixing zone during steady injection.
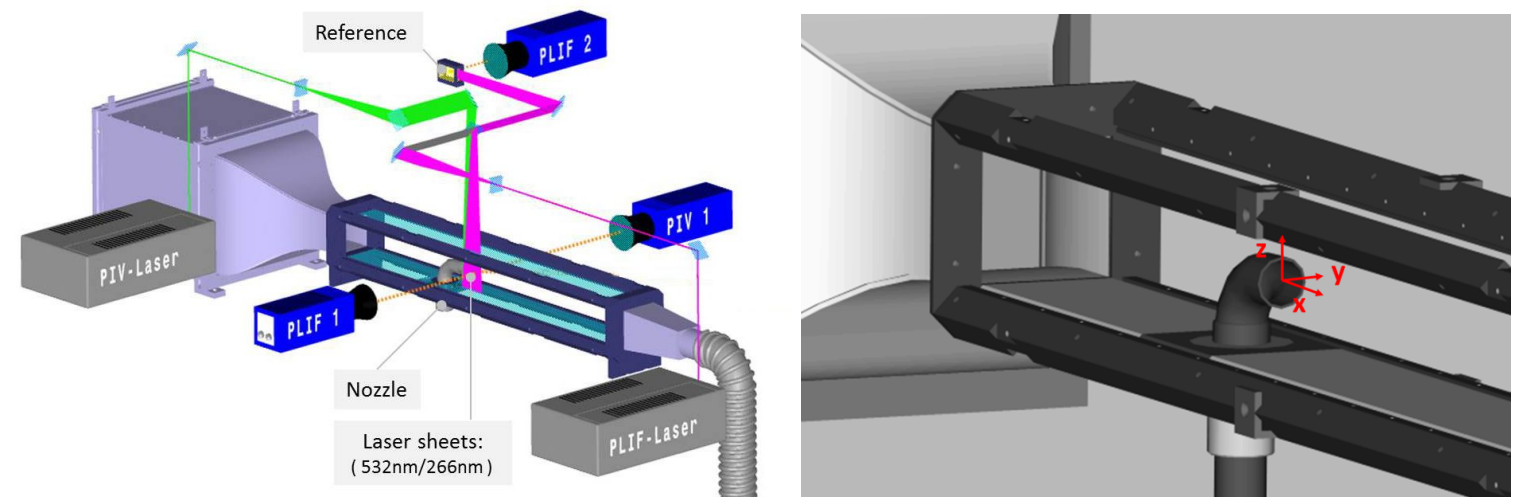

Figure 1: Schematic of the combined PLIF-PIV experimental setup (left) for measurements in the $\mathrm{X}-\mathrm{z}$ plane of the nozzle coordinate system (right) 
Turbulence, Heat and Mass Transfer 8

\section{Operation Conditions}

The wind tunnel is operated in suction mode and has a $830 \mathrm{~mm}$ long test section with a square cross-section of internal width of $76 \mathrm{~mm}$. Quartz windows with $45^{\circ}$ edge bevels provide optical access to the entire cross-section. Reproducible turbulent flow conditions are provided by a turbulence grid made of perforated steel that is placed immediately upstream of the test section at the exit of contraction nozzle. The turbulent fluctuations of the main flow immediately upstream of the mixing port have decayed to levels of $T u=0.02$ which is verified by planar PIV measurements at center plane.

\section{Combined Acetone PLIF/PIV Setup}

The optical setup for the combined PLIF-PIV measurements is depicted in Fig. 1. Instantaneous concentration measurements are implemented using planar laser induced fluorescence (PLIF) of the acetone seeded injected flow following the work of [2], [3], [4]. The Acetone is excited with a quadrupled Nd:YAG pulse laser at $266 \mathrm{~nm}$. Images of the acetone fluorescence are acquired with an intensified camera (Dicam-Pro, PCO AG) equipped with a S20Q photocathode and a Nikkor $50 / f_{\#} 1.2$ lens. After $2 \times 2$ binning the spatial resolution is 6 pixel $/ \mathrm{mm}$. Simultaneous recording of the PLIF Laser sheet intensity profile allows to determine the instantaneous excitation intensity profile thereby enabling shot-to-shot intensity normalization [1].

Simultaneous planar 2-C velocity measurements are obtained using particle image velocimetry (PIV) in a conventional orthogonal viewing arrangement. The dual frame sCMOS camera (ILA.sCMOS.PIV, ILA GmbH) observes the field of view at a magnification of approximately $22 \mathrm{pixel} / \mathrm{mm}$. The paraffin tracer droplets are illuminated using a doubled Nd:YAG laser with a rated pulse energy of $50 \mathrm{~mJ}$ (Brilliant Twins, Quantel/BigSky Laser). For the experiments at elevated jet temperatures, the injection port is seeded using a Laskin atomizer filled with a highly refined hydrocarbon mineral oil (Vicount Smoke Oil 135/180) which has a higher boiling temperature than paraffine oil which tended to evaporate prior to injection into the co-flow. In comparison the scattering diameter of the oil droplets at the mixing port outlet at $\mathrm{T}=393 \mathrm{~K}$ $(\Delta \mathrm{T}=100 \mathrm{~K})$ is still large enough to provide a sufficiently high PIV signal.

\section{Results}

\subsection{Pulsed Isothermal Injection}

Fig. 2 shows single shots of the mass fraction during pulsed operation in the $x-z$ center plane: (1) Beginning of injection,(2) strong injection and asymmetric vortex growing on the edges,(3) flow breakdown leaving behind small vortices and (4) again strong injection and forming of a large asymmetric vortex ring.

Phase averaging of single shot PLIF-PIV measurements in the $x-z$ center plane gives a better insight into the temporal shear layer and vortex evolvement during a pulsation cycle. Phase sorting of the single shot measurements is performed on the data using 36 equidistant bins $\left(10^{\circ}\right.$ bin size). Fig. 3 shows phase averaged velocity and concentration fields at the beginning of injection. At $\left(\Phi=320^{\circ}\right)$ small vortices occurs immediately upstream of the nozzle exit. At $\left(\Phi=340^{\circ}\right)$ the outer vortex has moved almost twice the distance as the inner vortex which leads to a faster vortex and higher growth due to entrainment. This effect seems to support stronger mixing and diffusion in the outer shear layer in comparison to the inner shear layer. 


\section{J. Klinner et al.}

\subsection{Elevated Jet Temperature}

The increased jet temperature leads to higher velocities within the jet due to decreasing air density at the given mass flow rate. This is confirmed by PIV measurements of the axial velocity in the $x-y$ center plane are presented in Fig. 4.

In Fig. 5, mass fraction profiles at three different jet temperatures are presented. Upstream at $x=8.4 \mathrm{~d}$, the increasing jet temperature leads to a higher mixing and diffusion. The near wall mass fraction at a distance of $\mathrm{x}=8.4 \mathrm{~d}$ is increasing from 0.25 (isothermal) to $0.30(\Delta \mathrm{T}=50 \mathrm{~K}$ ) and finally reaches 0.35 at $\Delta \mathrm{T}=100 \mathrm{~K}$ while the mass fraction on center line (nozzle axis) stays almost constant at all temperatures. Unique center line mass fractions are confirmed from measurements in the orthogonal $x-z$ center plane. At the most upstream distance the integral mass fraction of each profile is different which makes it difficult to compare measurements. Reasons could include secondary flow effects, different initial densities of the injected air and increasing densities while the hot jet mixes with cold air from the inlet. The influence of density changes on the accuracy of mass fraction measurements requires further investigations.

\section{References}

1. J. Klinner, V. Mayer, J. Heinze, and C. Willert. Simultaneous measurements of mixing fraction and velocities of a coaxial jet in a turbulent channel flow. In 17th Int Symp on Applications of Laser Techniques to Fluid Mechanics, 2014.

2. A. Lozano, B. Yip, and R.K. Hanson. Acetone: a tracer for concentration measurements in gaseous flows by planar laser-induced fluorescence. Experiments in Fluids, 13(6):369-376, 1992.

3. R. A. Bryant, J. M. Donbar, and J. F. Driscoll. Acetone laser induced fluorescence for low pressure/low temperature flow visualization. Experiments in Fluids, 28(5):471-476, 2000.

4. Mark C. Thurber, Frédéric Grisch, Brian J. Kirby, Martin Votsmeier, and Ronald K. Hanson. Measurements and modeling of acetone laser-induced fluorescence with implications for temperatureimaging diagnostics. Appl. Opt., 37(21):4963-4978, Jul 1998.

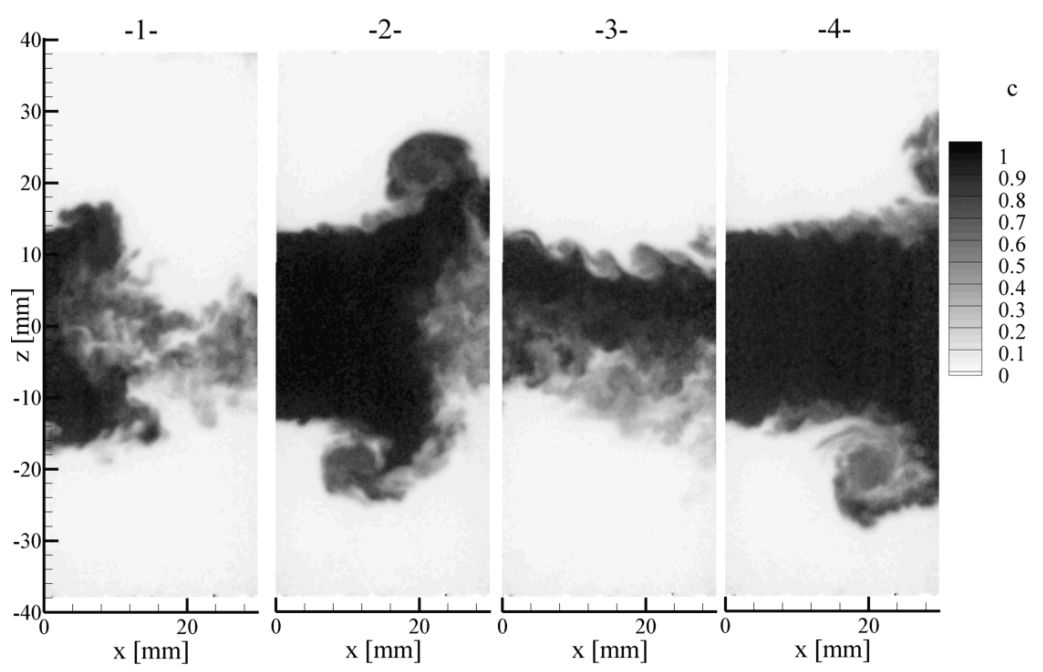

Figure 2: Snapshots of the concentration distribution in the $x-z$ center plane near the bend exit with pulsed injection (pulsation frequency $66 \mathrm{~Hz}$, acquisition frequency $5 \mathrm{~Hz}$ ) 

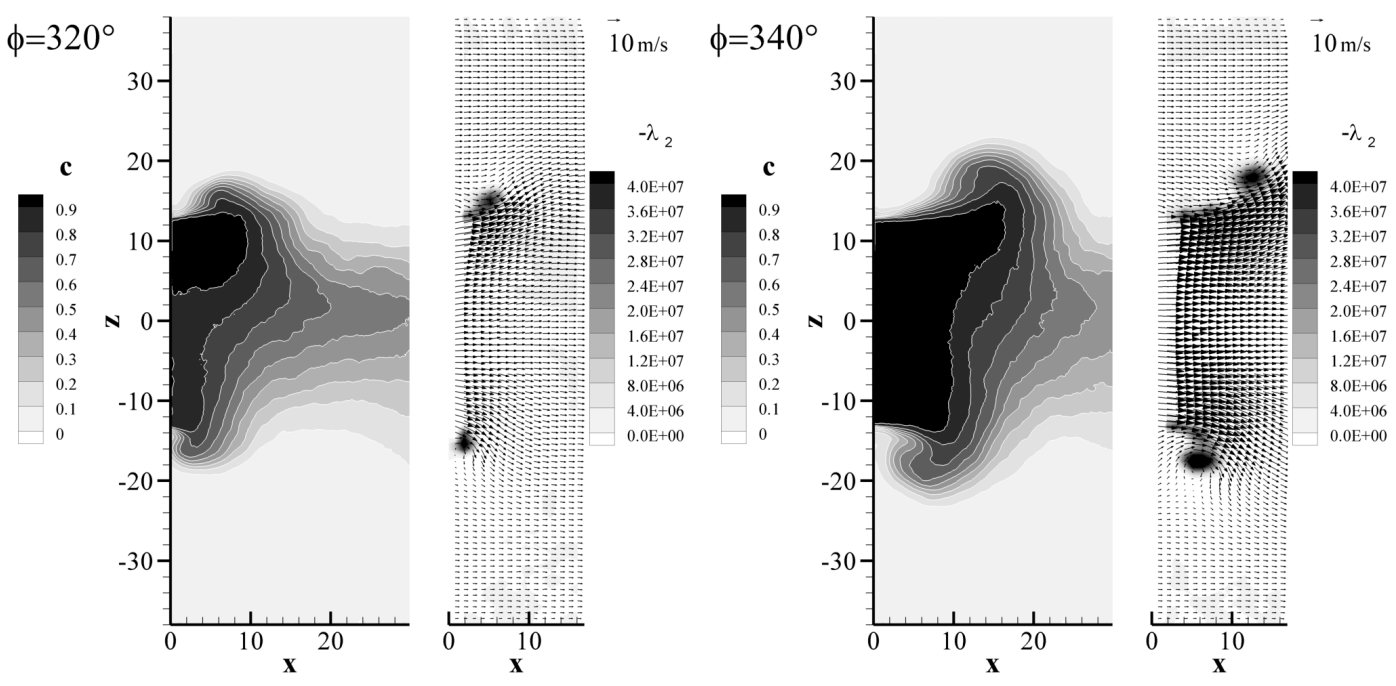

Figure 3: Phase averaged concentration and velocity fields in the $x-z$ center plane at the beginning of injection (bin size $=10^{\circ}$, approx. 100 shots per bin)
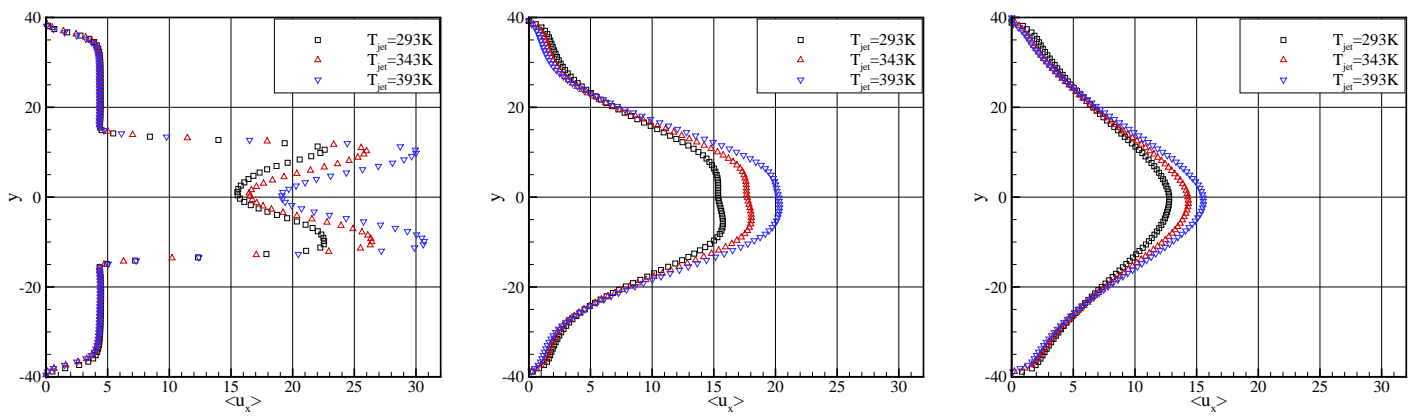

Figure 4: Axial velocity at increasing jet temperatures in the in the $x-y$ center plane: $x=0.4 d$ (left), $x=4.4 d$ (middle) and $x=8.4 d$ (right); $T_{\text {inlet }}=293 \mathrm{~K}$
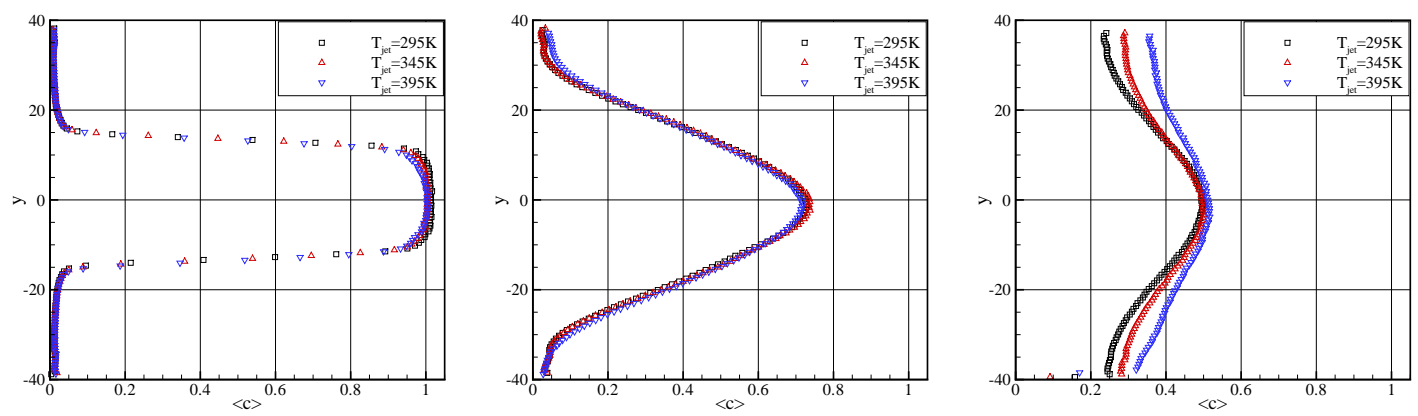

Figure 5: Mass fraction at increasing injection temperatures in the in the $x-y$ center plane: $x=0.4 d$ (left), $x=4.4 d$ (middle) and $x=8.4 d$ (right) 